\title{
THE COMBINATORICS OF CERTAIN PRODUCTS
}

\author{
DON RAWLINGS
}

ABSTRACT. A combinatorial interpretation for the coefficients in the expansion of $\Pi\left(1+u x^{j} y^{k}\right)\left(1-u x^{j} y^{k}\right)^{-1}$ is given.

1. Introduction. The coefficients $A(n ; x, y), B(n ; x, y)$ and $C(n ; x, y)$ defined by

$$
\begin{gathered}
\sum_{n \geqslant 0} \frac{A(n ; x, y) u^{n}}{(x)_{n}(y)_{n}}=\frac{1}{(u ; x, y)}, \\
\sum_{n \geqslant 0} \frac{B(n ; x, y) u^{n}}{(x)_{n}(y)_{n}}=(-u ; x, y), \\
\sum_{n \geqslant 0} \frac{C(n ; x, y) u^{n}}{(x)_{n}(y)_{n}}=\frac{(-u ; x, y)}{(u ; x, y)},
\end{gathered}
$$

where

$$
\begin{gathered}
(x)_{n}=(1-x)\left(1-x^{2}\right) \cdots\left(1-x^{n}\right), \quad(x)_{0}=1, \\
(u ; x, y)=\prod_{j, k \geqslant 0}\left(1-u x^{j} y^{k}\right),
\end{gathered}
$$

have been considered by a number of mathematicians. Carlitz [2] was the first to demonstrate that these coefficients are polynomials in $x$ and $y$ with positive integral coefficients and provided closed formulas for calculating them. In a subsequent paper Roselle [6] gave combinatorial interpretations for $A(n ; x, y)$ and $B(n ; x, y)$. More recently, generalizations of (1.1) and (1.2) have appeared in $[3,4,5]$ and $[4]$, respectively.

A natural question, which was suggested to me by Dominique Foata, arises from Roselle's work: Is there a combinatorial interpretation for $C(n ; x, y)$ ? This note provides one in terms of the already known interpretations of $A(n ; x, y)$ and $B(n ; x, y)$ and in terms of certain statistics defined on bicolored permutations.

2. Roselle's work. The polynomials $A(n ; x, y)$ and $B(n ; x, y)$ may be interpreted as the generating functions for various statistics defined on the group $G(n)$ consisting of permutations of $\{1,2, \ldots, n\}$. To be precise, if the major index of a permutation $\sigma \in G(n)$, denoted $m(\sigma)$, is defined to be the sum of the elements in the set

$$
\{j: \sigma(j)>\sigma(j+1), 1 \leqslant j \leqslant n-1\}
$$

Received by the editors September 30, 1982.

1980 Mathematics Subject Classification. Primary 05A15.

(C)1983 American Mathematical Society $0002-9939 / 83 \$ 1.00+\$ .25$ per page 
and the $i$-major and co-i-major indices are defined, respectively, to be

$$
\begin{gathered}
i(\sigma)=m\left(\sigma^{-1}\right), \\
c(\sigma)=\left(\begin{array}{c}
n \\
2
\end{array}\right)-i(\sigma),
\end{gathered}
$$

then, as Roselle [6] and Foata [4] have demonstrated,

$$
\begin{aligned}
& A(n ; x, y)=\sum_{\sigma \in G(n)} x^{m(\sigma)} y^{i(\sigma)}, \\
& B(n ; x, y)=\sum_{\sigma \in G(n)} x^{m(\sigma)} y^{c(\sigma)} .
\end{aligned}
$$

3. Bicolored permutations. Since Carlitz [2] showed that $C(n ; 1,1)=2^{n} \cdot n$ !, it is clear that $G(n)$ will not provide a combinatorial structure for interpreting $C(n ; x, y)$. For this reason, one is led to consider the set of bicolored permutations $B G(n)$, consisting of words $b=b(1) b(2) \cdots b(n)$ obtained by coloring each letter of some permutation $\sigma=\sigma(1) \sigma(2) \cdots \sigma(n) \in G(n)$ either red (indicated by underlining the letter) or blue (letter not underlined). For instance,

$$
b=3 \underline{2} 7 \underline{4} \underline{1} \underline{6} 5 \in B G(7) .
$$

Note that there are $2^{n}$ different colorings of each $\sigma \in G(n)$.

Now, possessing a combinatorial set with the correct cardinality, all that remains is to define some appropriate statistics on $B G(n)$ for which $C(n ; x, y)$ is the generating function. To this end, let $W(n)$ be the set of words $w=w(1) w(2) \cdots w(n)$ of length $n$ with letters $w(i) \in\{0,1\}$, and let $|w|$ denote the number of letters equal to 1 in $w$. The reduction of a permutation $\gamma$ of $\left\{a_{1}<a_{2}<\cdots<a_{n}\right\}$ is obtained by replacing $a_{i}$ in $\gamma$ by $i$ for $1 \leqslant i \leqslant n$. For instance, the reduction of the permutation $\gamma=2416$ of $\{1<2<4<6\}$ is 2314 .

Each $b \in B G(n)$ is now assigned to a 4-tuple $(w, v, \theta, \alpha)$ where $w, v \in W(n)$ with $|w|=|v|, \theta \in G(|w|)$ and $\alpha \in G(n-|w|)$ according to the following rules:

$$
\begin{gathered}
w(i)= \begin{cases}1 & \text { if } b(i) \text { is red, } \\
0 & \text { otherwise, }\end{cases} \\
v(i)= \begin{cases}1 & \text { if } i \text { is red in } b, \\
0 & \text { otherwise, }\end{cases} \\
\theta=\text { reduction of the red subpermutation of } b, \\
\alpha=\text { reduction of the blue subpermutation of } b .
\end{gathered}
$$

Roughly speaking, $w$ indicates which positions of $b$ are red, $v$ which elements of $\{1,2, \ldots, n\}$ are placed in the red positions, $\theta$ how the red letters are arranged in the red positions, and $\alpha$ how the blue letters are placed in the remaining positions. For instance, the bicolored permutation $b$ of (3.1) corresponds to the 4-tuple $(w, v, \theta, \alpha)$ where $w=0101110, v=1101010, \theta=2314$ and $\alpha=132$. Note that the map $b \rightarrow(w, v, \theta, \alpha)$ is a bijection between $B G(n)$ and the set of such 4-tuples.

Finally, for the bicolored permutation $b \rightarrow(w, v, \theta, \alpha)$ the appropriate statistics are defined by

$$
\begin{gathered}
M(b)=m(\theta)+m(\alpha)+m(w), \\
I(b)=i(\theta)+c(\alpha)+m(v),
\end{gathered}
$$


where $m(w)$ and $m(v)$ are defined in exactly the same way as the major index of a permutation.

4. The interpretation of $C(n ; x, y)$. It is now possible to show that

$$
C(n ; x, y)=\sum_{b \in B G(n)} x^{M(b)} y^{I(b)} .
$$

First, as Carlitz [2] demonstrated, identities (1.1)-(1.3) imply

$$
C(n ; x, y)=\sum_{k \geqslant 0}\left(\begin{array}{l}
n \\
k
\end{array}\right)_{x}\left(\begin{array}{l}
n \\
k
\end{array}\right)_{y} A(k ; x, y) B(n-k ; x, y),
$$

where the $x$-binomial coefficient is defined by

$$
\left(\begin{array}{l}
n \\
k
\end{array}\right)_{x}=\frac{(x)_{n}}{(x)_{k}(x)_{n-k}} .
$$

Second, the fact that (see $[1, \mathrm{p} .40])$

$$
\sum x^{m(w)}=\left(\begin{array}{l}
n \\
k
\end{array}\right)_{x},
$$

where the summation is over $w \in W(n)$ with $|w|=k$, along with (2.4), (2.5), (3.6) and (3.7) allow the calculation

$$
\begin{aligned}
\sum_{b \in B G(n)} x^{M(b)} y^{I(b)} & =\sum_{(w, v, \theta, \alpha)} x^{m(\theta)+m(\alpha)+m(w)} y^{i(\theta)+c(\alpha)+m(v)} \\
& =\sum_{k \geqslant 0} \sum_{|w|=k} x^{m(w)} \sum_{|v|=k} y^{m(v)} \sum_{\theta \in G(k)} x^{m(\theta)} y^{i(\theta)} \sum_{\alpha \in G(n-k)} x^{m(\alpha)} y^{c(\alpha)} \\
& =\sum_{k \geqslant 0}\left(\begin{array}{l}
n \\
k
\end{array}\right)_{x}\left(\begin{array}{l}
n \\
k
\end{array}\right)_{y} A(k ; x, y) B(n-k ; x, y) .
\end{aligned}
$$

Identities (4.2) and (4.5) imply (4.1).

\section{REFERENCES}

1. G. E. Andrews, The theory of partitions, Addison-Wesley, Reading, Mass., 1976.

2. L. Carlitz, The expansion of certain products, Proc. Amer. Math. Soc. 7 (1956), 558-564.

3. A. M. Garsia and I. Gessel, Permutation statistics and partitions, Adv. in Math. 31 (1979), 288-305.

4. D. Foata, Aspects combinatoires du calcul des q-séries (Séminaire d'Informatique Théorique, 1980), Institut de Programmation, Centre National Recherche Scientifique No. 248, Paris, 1980.

5. D. Rawlings, Generalized Worpitzky identities with applications to permutation enumeration, European J. Combin. 2 (1981), 67-78.

6. D. P. Roselle, Coefficients associated with the expansion of certain products, Proc. Amer. Math. Soc. 45 (1974), 144-150.

Department of Mathematics, California Polytechnic State University, San luis Obispo, CALIFORNIA 93407 